\title{
Evaluating severe hypercholesterolemia in an uninsured, vulnerable population receiving care at a community clinic
}

\section{Josh Peedikayil}

The University of Texas Southwestern Medical Center

Clark Measom

The University of Texas Southwestern Medical Center

Brianna Wilson

The University of Texas Southwestern Medical Center

\section{Whitney Stuard}

The University of Texas Southwestern Medical Center

\section{Angel Valencia}

The University of Texas Southwestern Medical Center

\section{Ashlyn Lafferty}

The University of Texas Southwestern Medical Center

\section{Reena Jasani}

The University of Texas Southwestern Medical Center

\section{Cat Ahmed}

FH Foundation

\section{Marcus Hurt}

The University of Texas Southwestern Medical Center

\section{Amit Khera}

The University of Texas Southwestern Medical Center Nora Gimpel

The University of Texas Southwestern Medical Center

Zahid Ahmad ( $\nabla$ zahid.ahmad@utsouthwestern.edu ) The University of Texas Southwestern Medical Center

\section{Research Article}

Keywords: Familial Hypercholesterolemia, community health, statins, LDL-C, hyperlipidemia, uninsured

Posted Date: January 25th, 2022

DOI: https://doi.org/10.21203/rs.3.rs-1174772/v1 
License: (c) (i) This work is licensed under a Creative Commons Attribution 4.0 International License. Read Full License 


\section{Abstract}

\section{Background}

Individuals with $\mathrm{LDL}-\mathrm{C} \geq 190 \mathrm{mg} / \mathrm{dL}$ (severe hypercholesterolemia) require treatment with high-intensity statins and should be evaluated for underlying causes including Familial Hypercholesterolemia (FH). The characteristics of patients with LDL-C $\geq 190 \mathrm{mg} / \mathrm{dL}$ has been reported in routine healthcare settings, but limited data exist about uninsured populations.

Objectives

To examine the scope of LDL-C $\geq 190 \mathrm{mg} / \mathrm{dL}$ in a clinic serving uninsured populations.

Methods

Patients with LDL-C $\geq 190 \mathrm{mg} / \mathrm{dL}$ at North Dallas Shared Ministries, a charity, community clinic in Dallas, TX, were identified via query of the electronic health record, and data was extracted from medical charts. Patients were interviewed to obtain family history and assess knowledge of cholesterol levels.

Results

Among 662 patients with lipid measurements, 27 had LDL-C $\geq 190 \mathrm{mg} / \mathrm{dL}$ ( $67 \%$ female, 78\% Hispanic, mean age 52 years). Median pretreatment LDL-C was $210.5 \mathrm{mg} / \mathrm{dL}$. Almost all (93\%) were prescribed a statin, but only 33\% were prescribed high-intensity statin. Treated LDL-C was $141 \mathrm{mg} / \mathrm{dL}$. No patients had an ICD-10 diagnosis of FH, and only $31 \%$ had laboratory tests for secondary causes of hypercholesterolemia. Only $15 \%$ had any documentation of family history of hypercholesterolemia and/or ASCVD. After interviewing, we discovered previously undocumented family history of hypercholesterolemia in 54\% and ASCVD in 31\%.

\section{Conclusions}

Among the uninsured with LDL-C $\geq 190 \mathrm{mg} / \mathrm{dL}$ in a community clinic, the use of high-intensity statins was suboptimal, and most were not evaluated for causes of severe hypercholesterolemia. Future efforts to address gaps and education are warranted to improve care for severe hypercholesterolemia patients in this vulnerable population.

Condensed Abstract

Few studies evaluate severe hypercholesterolemia in uninsured populations. We queried the electronic health record at a charity community clinic to characterize individuals with severe hypercholesterolemia. Our results suggest more education is needed for patients and providers regarding severe hypercholesterolemia and $\mathrm{FH}$ to better care for this vulnerable population.

\section{Introduction}


Patients with severe hypercholesterolemia (defined as LDL-C levels $\geq 190 \mathrm{mg} / \mathrm{dL}$ ) have a high risk of developing premature atherosclerotic cardiovascular disease (ASCVD) and recurrent coronary events ${ }^{1-4}$. Clinical trial data have demonstrated that high intensity statins provide the greatest ASCVD risk reduction when compared to moderate-intensity statins or placebo for individuals with severe hypercholesterolemia and/or ASCVD 1,2,5,6. For this reason, guidelines recommend maximally tolerated statin therapy for patients with severe hypercholesterolemia with a goal of reducing low-density lipoprotein cholesterol (LDL-C) to below $100 \mathrm{mg} / \mathrm{dL}$, and such individuals should be evaluated for underlying causes of severe hypercholesterolemia including hypothyroidism, nephrotic syndrome, and cholestasis ${ }^{1,5}$.

Additionally, a significant portion of patients with LDL-C $\geq 190 \mathrm{mg} / \mathrm{dl}$ will have Familial Hypercholesterolemia $(\mathrm{FH})^{7}$. $\mathrm{FH}$ is an autosomal dominant condition characterized by a substantial elevation of $\mathrm{LDL}-\mathrm{C}^{8}$. Identifying and treating $\mathrm{FH}$ patients remains a top public health priority for effective ASCVD prevention ${ }^{8,9}$. In fact, the United States Centers for Disease Control and Prevention (CDC) designated $\mathrm{FH}$ as one of three Tier 1 genomic conditions requiring early detection and early intervention ${ }^{9,10}$.

While the characteristics of patients with LDL-C $\geq 190 \mathrm{mg} / \mathrm{dl}$ has been reported in routine healthcare systems, limited data exist about such individuals in vulnerable populations such as the medically underserved at charity, community clinics ${ }^{8,11}$. These clinics serve uninsured individuals who are unable to access care even from safety-net healthcare systems ${ }^{12}$. Furthermore, no data exists regarding the burden of $\mathrm{FH}$ and associated treatment gaps for this population. Here, we sought to begin addressing these gaps by describing the burden of severe hypercholesterolemia at a primary care, charity, community clinic.

\section{Methods}

\section{Setting and Participants}

The study was conducted at North Dallas Shared Ministries (NDSM) and was approved by the Institutional Review Board at UT Southwestern Medical Center. NDSM is a cooperative effort of 47 congregations that combine resources to deliver assistance to the poorest families and individuals in Dallas, TX. Among its many programs, NDSM includes a charity, community clinic that provides care to uninsured populations. The clinic first opened in 2000 and grew rapidly to become a full-time primary care clinic, now open 7 AM - 7 PM weekdays. As of 2019, over 7000 medically underserved individuals receive routine health care at NDSM annually ${ }^{13}$. These patients are uninsured and are unable to receive routine healthcare from safety-net healthcare systems. The electronic health records (EHR) used at NDSM is Athena Electronic Health Records (Watertown, MA) since $2016^{14}$.

\section{Data Collection}


We queried the EHR at NDSM for patients who met our eligibility criteria since 2016 (when HER was implemented at NDSM). Inclusion criteria included any recorded LDL-C $\geq 190 \mathrm{mg} / \mathrm{dL}$. We did not exclude any individuals among those with LDL-C $\geq 190 \mathrm{mg} / \mathrm{dL}$. The charts were manually reviewed to collect information on demographics, past medical and family medical history, untreated and treated LDL-C levels, comorbidities, and use of lipid lowering drugs. Physical exam findings were reviewed for documentation of arcus senilis and xanthomas (for $\mathrm{FH}$ ). We searched charts (both clinic notes and laboratory results) for evidence of secondary causes of severe hypercholesterolemia, including hypothyroidism (Thyroid Stimulating Hormone (TSH) level), cholestasis (bilirubin), and nephrotic syndrome (urine protein). For those without evidence of secondary causes, we sought to establish whether they fulfill FH diagnostic criteria for Simon Broome and/or Dutch Lipid Clinic Network (DLCN) 15,16 (Appendix A and B). Additionally, Make Early Diagnosis to Prevent Early Dealths (MEDPED) criteria was used to determine the FH status of patients using age and lipid levels ${ }^{17,18}$ (Appendix C).

All charts that met inclusion criteria were reviewed by JP and BW, with supervision by a University of Texas Southwestern (UTSW) FH clinic physician, ZA. Study data was stored within the Research Electronic Data Capture (REDCap) database and the study was approved by the UTSW Institutional Review Board.

\section{Patient Interviews}

Patients who met qualification criteria were called via telephone for two purposes: we gathered further past medical and family history (not previously recorded EHR) needed to assess whether they fulfill Simon Broome and/or DLCN FH diagnostic criteria; and patients were asked a series of questions about their knowledge of cholesterol and hypercholesterolemia (Appendix D). The interviews were conducted in the patient's native language by fluent interviewers. All patients were free to answer or not answer any question that was posed to them.

\section{Statistics}

Continuous variables were summarized as means with standard deviation and/or medians with associated interquartile ranges (IQR's). Categorical variables were summarized with percentages. The interviews with patients were conversational and from the information gathered, summary statistics were created.

\section{Results}

\section{Study Population}

Between 2016 and 2020, LDL-C values were measured for 662 unique patients at NDSM clinic. Twentyseven of these patients met inclusion criteria, having at least one measured LDL-C $\geq 190 \mathrm{mg} / \mathrm{dL}$ (Table 1). Population mean age was 52 years with a range of 38-79 years as well as a female predominance (67\%). Most patients self-identified as Hispanic (74\%) and white (63\%), which reflects the proportion at 
the NDSM clinic overall. Sixty-three percent of patients also had hypertension and 30\% had also been diagnosed with type 2 diabetes mellitus. Three patients (11\%) had hypothyroidism, a possible secondary cause of hypercholesterolemia, but over half (59\%) did not have all the laboratory tests needed to exclude secondary causes of severe hypercholesterolemia (TSH, urine protein, and/or bilirubin).

\section{Lipid levels}

Of the 27 patients who met inclusion criteria, 26 were untreated LDL-C levels, 21 patients also had treated LDL-C levels available (Figure 1), and six had multiple measurements of LDL-C $\geq 190 \mathrm{mg} / \mathrm{dL}$. The mean time period between pre- and post-treatment lipid measurements was 0.98 years.

Mean pre-treatment total cholesterol levels (standard deviation) were $300.9 \mathrm{mg} / \mathrm{dL}$ (21.7) and 219.6 $\mathrm{mg} / \mathrm{dL}$ (45.4) after treatment. LDL-C levels were $213.6 \mathrm{mg} / \mathrm{dL}$ (18.2) before treatment and $137.4 \mathrm{mg} / \mathrm{dL}$ (35) post-treatment. HDL-C pre-treatment was $48.5 \mathrm{mg} / \mathrm{dL}$ (13.2) and $49 \mathrm{mg} / \mathrm{dL}$ (11.8) post-treatment. Median triglyceride levels (range) were $207 \mathrm{mg} / \mathrm{dL}$ (88 - 375) pre-treatment and $163 \mathrm{mg} / \mathrm{dL}$ (64 - 460) post-treatment (Table 1).

\section{Statin prescriptions}

Almost all patients (93\%) were prescribed a statin medication (Figure 2). After treatment, $14 \%$ of patients achieved LDL-C levels below $100 \mathrm{mg} / \mathrm{dL}$ and 10\% had LDL-C levels that remained above $190 \mathrm{mg} / \mathrm{dL}$.

Only $33 \%$ were prescribed a high intensity statin, defined as atorvastatin $40-80 \mathrm{mg}$ or rosuvastatin $20-40$ $\mathrm{mg}$ daily. Most patients that were prescribed atorvastatin were only taking 10-20 mg daily. Other statins prescribed included simvastatin (15\%) and lovastatin (26\%). Two patients had not been prescribed a statin medication, relying solely on lifestyle modification per EHR provider notes.

\section{Family history}

Documentation about family history was sparse, with $19 \%$ having any documentation of hypercholesterolemia in their family and 15\% having any documentation of ASCVD in their family.

\section{FH diagnosis}

No patients had an ICD-10 diagnosis of FH, and no patients had any mention of FH or FH family screening anywhere within the medical record.

Because of missing family history documentation, determining $\mathrm{FH}$ status from the Simone Broome or DLCN criteria, which includes family history, was incomplete. No patients fulfilled criteria for "Definite FH" for either Simon Broome or DLCN. One patient met criteria for "possible FH" for the Simone Broome Criteria. All 27 patients met DLCN criteria for "possible FH." Using MEDPED, no patients met criteria for FH diagnosis.

\section{Patient interviews}


Of the 27 patients (8 English speakers, 19 Spanish speakers) that met inclusion criteria, we were able to interview 13 (4 English speakers, 9 Spanish speakers) (Table 2). Over half (54\%) of the patients had family history of high cholesterol and $31 \%$ had a family history of cardiovascular disease, neither of which were previously documented in the EHR. With this new information, nine (69\%) now met criteria for "Possible FH" based on the Simone Broome criteria.

Approximately half (54\%) of the patients claimed to have never heard of the terms LDL-C (low density lipoprotein-cholesterol) and HDL-C (high density lipoprotein-cholesterol). The patients that had heard of these terms commonly understood them as "one is bad, and one is good" however being unsure which was which. Almost all (92\%) patients could recall being told they had high cholesterol by a health care provider either at NDSM or another clinic. Additionally, $15 \%$ of patients recalled hearing the term "Familial Hypercholesterolemia" but no patients could give a detailed description of the disease.

All patients reported knowing the importance of lowering cholesterol levels, citing reasons such as "eliminating heart attack and stroke" and "to live... [not wanting] to die." In addition, 92\% cited ways that they knew to lower cholesterol including "exercise, eating properly, less stress" and "staying away from certain [fried] foods", but only one patient stated taking medication as an action that could be taken.

Of the 13 patients contacted, only 4 reported currently adhering to statin therapy. The reasons given by patients for not being on statin therapy ranged from intolerance (headaches, cramps), difficulty in obtaining prescription refills, and patients stopping medication after their cholesterol levels "normalized" (Table 3).

Table 1. Demographics and characteristics of patients with LDL-C $\geq 190 \mathrm{mg} / \mathrm{dL}$ obtained from EHR at NDSM clinic. 


\begin{tabular}{|ll}
\hline Characteristic & Value $(\mathbf{n}=\mathbf{2 7})$ \\
\hline Age (years), mean (range) & $52(38-79)$ \\
\hline Female, $\mathbf{n}(\%)$ & $18(67)$ \\
\hline Ethnicity, $\mathbf{n}(\%)$ & $20(74)$ \\
\hline Hispanic & $5(19)$ \\
\hline Non-Hispanic & $2(7)$ \\
\hline Unknown & \\
\hline Race, $\mathbf{n}(\%)$ & $5(19)$ \\
\hline Black/ African American & $17(62)$ \\
\hline Caucasian & $5(19)$ \\
\hline Unknown & $8(30)$ \\
\hline Past Medical History, $\mathbf{n}(\%)$ & $17(63)$ \\
\hline Diabetes & $3(11 \%)$ \\
\hline Hypertension & $8(30)$ \\
\hline Hypothyroidism &
\end{tabular}

\section{Family History: Hypercholesterolemia, n (\%)}

$\begin{array}{ll}\text { Yes } & 1(4) \\ \text { No } & 4(15) \\ \text { Not Documented } & 22(81)\end{array}$

\section{Family History: ASCVD, $\mathrm{n}(\%)$}

$\begin{array}{ll}\text { Yes } & 0(0) \\ \text { No } & 4(15) \\ \text { Not Documented } & 23(85)\end{array}$

Total Cholesterol, median (range) $\mathrm{mg} / \mathrm{dL}$

Pre-Treatment*

Post-Treatment†
301 (254-354)

207 (160-324)

LDL-Cholesterol, median (range) $\mathrm{mg} / \mathrm{dL}$

Pre-Treatment* 
Post-Treatment†

$141(92-211)$

HDL-Cholesterol, median (range) $\mathrm{mg} / \mathrm{dL}$

Pre-Treatment*

$48(28-85)$

Post-Treatment†

$45(34-72)$

Triglycerides, median (range) $\mathrm{mg} / \mathrm{dL}$

Pre-Treatment*

207 (88-375)

Post-Treatment†

$163(64-460)$

*Pre-Treatment $\mathrm{n}=26$

tPost-Treatment $\mathrm{n}=21$

Table 2. Responses of patients with LDL cholesterol $\geq 190 \mathrm{mg} / \mathrm{dL}$ in a charity, community clinic

\begin{tabular}{lll} 
& \# of patients & $\%$ of patients \\
\hline Total patients interviewed & 13 & \\
\hline Yes, family history of high cholesterol & 7 & $54 \%$ \\
\hline Yes, family history of heart disease & 4 & $31 \%$ \\
\hline Yes, current or remote tobacco use & 5 & $38 \%$ \\
\hline Unfamiliar with the terms "LDL" and "HDL" & 7 & $54 \%$ \\
\hline Remember being told that they have high cholesterol & 12 & $92 \%$ \\
\hline Currently taking a statin drug & 4 & $31 \%$ \\
\hline Familiar with the term “Familial Hypercholesterolemia" & 2 & $15 \%$ \\
\hline Claim they know how to lower their cholesterol & 12 & $92 \%$ \\
\hline Believe lowering cholesterol is important for their health & 13 & $100 \%$
\end{tabular}

Table 3: Selected patient quotes related to barriers to care, taken from phone interviews of patients with $\mathrm{LDL}$ cholesterol $\geq 190 \mathrm{mg} / \mathrm{dL}$ in a charity, community clinic 
Statin "I have taken different [statins] before. I stopped taking some because it gave me intolerance cramps in my legs."

“...they put me on statins of $10 \mathrm{mg}$. The $20 \mathrm{mg}$ make me feel funny. I don't know what it is, all my joints were sore, so I had to get off it. [...] I took the pills, but I think I need to go back to the $10 \mathrm{mg}$. The 20 and 40 were too much for me."

Care gaps "I also can't get the prescriptions enough. Being a trucker, it's hard to eat right and do what you need to do."

"My mama died of a massive heart attack at 39. My brother also just had a triple bypass."

Patient "[l can treat my cholesterol by] tak[ing] cholesterol medicine and eat[ing] less fat, education sugar, carbohydrates and flour."

"There is one, [HDL or LDL] that is good and one that is bad, but I don't remember which one is which."

Lowering cholesterol is important "because it can clog arteries."

"[elevated cholesterol] can give me a heart attack, it can raise my blood pressure, and it can cause thrombosis."

"I have heard of HDL and LDL, but they were not explained in the clinic."

\section{Discussion}

In an uninsured population receiving care at a charity community clinic, we identified several gaps: only $10 \%$ of all patients had had lipid levels measured; few patients with severe hypercholesterolemia were on high intensity statin drugs; and family history was missing in nearly all patients as was laboratory workup for secondary causes of severe hypercholesterolemia. FH was not mentioned in any of their medical records. Subsequently, after calling the severe hypercholesterolemia patients to gather more information, many had previously unknown family histories of premature ASCVD and hypercholesterolemia.

While many studies have looked at $\mathrm{FH}$ and severe hypercholesterolemia within large health systems, prior studies have not looked at the burden of disease at a charity, community clinic serving underserved, uninsured populations who are unable to access care even from safety-net healthcare systems ${ }^{12,19,20}$. An estimated 26 million uninsured patients rely on one of 1,400 charity, community clinics across the United States ${ }^{21}$. Such patients suffer from disparities in care, often getting fewer treatments or treatments not adherent to guidelines, and the uninsured have higher morbidity and mortality relative to the general population ${ }^{21}$.

Our findings at NDSM are consistent - albeit more pronounced - with patient cohorts in various, large health care systems. Within our study, the population mean age of 52 and female predominance $(67 \%)$ are similar to the demographics of previous studies of patients with severe hypercholesterolemia and 
possible $F H^{20,22,23}$. At NDSM, only $33 \%$ were prescribed a high-intensity statin compared to $42 \%$ across patients in the CASCADE-FH registry which was conducted across 11 different US lipid clinics ${ }^{20}$. In addition, the treated LDL-C levels with statin therapy were higher in our study than comparative studies both in the US and abroad. ${ }^{20,23-25}$

In our study population, $30 \%$ of patients were previously diagnosed with Type II Diabetes Mellitus (T2DM). This is a higher rate than the national age adjusted prevalence of $13.0 \%$ and much higher than other cohorts of patients with severe hypercholesterolemia and $\mathrm{FH}^{20,26}$. Further, studies published by Pijlman et al. in the Netherlands and Climent et al. using the Dyslipidemia Registry of the Spanish Arteriosclerosis Society have both found that cohorts of patients with mutation positive $\mathrm{FH}$ have a decreased prevalence of T2DM than the general population and those with severe hypercholesterolemia 24,27 . Based on our findings, there are a few possibilities for this discrepancy. First, our cohort of patients has not been genetically tested for FH mutations and this is in line with findings from the Spanish Arteriosclerosis Society that patients with suspected FH have a prevalence of T2DM more similar to the general population ${ }^{27}$. Additionally, decreased access to care in this patient population could result in the increased prevalence of T2DM when compared to patients with severe hypercholesterolemia described in other studies. One of the contributing factors to the decreased prevalence of T2DM in the FH population is the assumption that patients who have been screened and diagnosed early with $\mathrm{FH}$ are more likely to have routine, aggressive care at an earlier age, leading to a generally healthier lifestyle ${ }^{27}$. This does not fit the context of our patient population at NDSM as these patients have not been diagnosed with FH and have not been treated as aggressively as severe hypercholesterolemia patients in other medical systems. This along with the increased rate of type 2 diabetes in the uninsured population compared to insured may explain this difference ${ }^{28}$.

In our limited interviews, we were able to elicit issues affecting hypercholesterolemia treatment and medication adherence from the perspective of patients. These gaps in care and intolerance to medications are similar to barriers to FH care identified by Jones et al. in their interviews with patients diagnosed with Familial Hypercholesterolemia in a large healthcare system ${ }^{29}$. However, Jones et al. did not identify inability to get prescriptions as a barrier to $\mathrm{FH}$ patient care ${ }^{29}$. We believe this may be due to the higher number of uninsured patient's that are treated at NDSM relative to the $97 \%$ insured patient population that was interviewed by Jones et al. Although the reasons for provider under prescribing and patient nonadherence are complex, these anecdotes perhaps give insight into specific opportunities at charity, community clinics and may be an area of improvement.

Our findings suggest that there is not enough knowledge about $\mathrm{FH}$ and severe hypercholesterolemia in the patient population as well as from providers. Future efforts should address these issues, with patient education on hypercholesterolemia within charity, community clinics as well as provider education about lipid screening and assessing patients with severe hypercholesterolemia.

\section{Limitations}


There are several limitations to the study. First, we were only able to obtain LDL-C values from patients at NDSM who had been given a lipid screening. While the 2018 AHA/ACC Cholesterol Guidelines recommends that all patients between the ages of 40-75 should be screened for lipid abnormalities annually, it is likely there are patients at the clinic with elevated LDL-C who have simply not been screened $1,9,30$. Because all patient data was taken from the NDSM Athena EHR, our study is limited to patients who had an untreated lipid screening after NDSM switched to Athena's EHR in 06/2016. Therefore, patients with LDL-C $\geq 190 \mathrm{mg} / \mathrm{dL}$ prior to this time who had been given a statin would likely not be represented in this study. Further, our study has all the limitations that come with limited sample size. A greater understanding of the populations knowledge of cholesterol could be gleamed if we were able to have a larger sample size and speak to a greater proportion of patients.

\section{Conclusions}

Among a medically underserved community with LDL-C $\geq 190 \mathrm{mg} / \mathrm{dL}$ in a charity community clinic, we found use of high intensity statins was suboptimal and most patients were not evaluated for underlying causes of severe hypercholesterolemia. Future efforts to improve care and education are warranted for both providers and uninsured patients.

\section{Abbreviations}

$\mathrm{FH}$

Familial Hypercholesterolemia.

ASCVD

Atherosclerotic cardiovascular disease

LDL-C

Low density lipoprotein-cholesterol

HDL-C

Low density lipoprotein-cholesterol

NDSM

North Dallas Shared Ministries

DLCN

Dutch Lipid Clinic Network

\section{Declarations}

Acknowledgements: None

- Funding: none

- Ethics approval: study was approved by UT Southwestern IRB. All experimental protocols were approved by the UT Southwestern Institutional Review Board. 
- Consent to participate: since the study involved retrospective review of de-identified data, no individual consent forms were signed by patients.

- Consent for publication: $\mathrm{n} / \mathrm{a}$

- All methods were carried out in accordance with relevant guidelines and regulations

- Availability of data and material: "The data that support the findings of this study are available from the corresponding author upon request."

- Code availability: $n / a$

- Authors contributions: All authors contributed to the study conception and design. Material preparation, data collection and analysis were performed by Josh Peedikayil, Clark Measom, Brianna Wilson, Whitney Stuard, Angel Valencia, and Ashlyn Lafferty. Writing - review and editing: Reena Jasani, Cat Davis Ahmed, Marcus Hunrt, Amit Khera, Nora Gimpel, and Zahid Ahmad. All authors read and approved the final manuscript.

\section{References}

1. Grundy SM, Stone NJ, Bailey AL, et al. 2018 AHA/ACC/AACVPR/AAPA/ABC/ACPM/ADA/AGS/APhA/ASPC/NLA/PCNA Guideline on the Management of Blood Cholesterol: A Report of the American College of Cardiology/American Heart Association Task Force on Clinical Practice Guidelines. J Am Coll Cardiol. 2019;73(24):e285-e350.

2. Virani SS, Alonso A, Aparicio HJ, et al. Heart Disease and Stroke Statistics-2021 Update: A Report From the American Heart Association. Circulation. 2021;143(8):e254-e743.

3. Zhang Y, Vittinghoff E, Pletcher MJ, et al. Associations of Blood Pressure and Cholesterol Levels During Young Adulthood With Later Cardiovascular Events. J Am Coll Cardiol. 2019;74(3):330-341.

4. Fernandez-Friera L, Fuster V, Lopez-Melgar B, et al. Normal LDL-Cholesterol Levels Are Associated With Subclinical Atherosclerosis in the Absence of Risk Factors. J Am Coll Cardiol. 2017;70(24):2979-2991.

5. Luirink IK, Wiegman A, Kusters DM, et al. 20-Year Follow-up of Statins in Children with Familial Hypercholesterolemia. N Engl J Med. 2019;381(16):1547-1556.

6. Goldberg AC, Hopkins PN, Toth PP, et al. Familial hypercholesterolemia: screening, diagnosis and management of pediatric and adult patients: clinical guidance from the National Lipid Association Expert Panel on Familial Hypercholesterolemia. J Clin Lipidol. 2011;5(3):133-140.

7. Singh S, Bittner V. Familial hypercholesterolemia--epidemiology, diagnosis, and screening. Curr Atheroscler Rep. 2015;17(2):482.

8. Representatives of the Global Familial Hypercholesterolemia C, Wilemon KA, Patel J, et al. Reducing the Clinical and Public Health Burden of Familial Hypercholesterolemia: A Global Call to Action. JAMA Cardiol. 2020;5(2):217-229.

9. Bucholz EM, Rodday AM, Kolor K, Khoury MJ, de Ferranti SD. Prevalence and Predictors of Cholesterol Screening, Awareness, and Statin Treatment Among US Adults With Familial 
Hypercholesterolemia or Other Forms of Severe Dyslipidemia (1999-2014). Circulation. 2018;137(21):2218-2230.

10. Abul-Husn NS, Manickam K, Jones LK, et al. Genetic identification of familial hypercholesterolemia within a single U.S. health care system. Science. 2016;354(6319).

11. Brett T, Qureshi N, Gidding S, Watts GF. Screening for familial hypercholesterolaemia in primary care: Time for general practice to play its part. Atherosclerosis. 2018;277:399-406.

12. Darnell J. What is the role of free clinics in the safety net? Med Care. 2011;49(11):978-984.

13. North Dallas Shared Ministries. 2019 Annual Report. In:2019.

14. Athena Health Headquarters. https://www.athenahealth.com/about/contact-us. Published 2021. Accessed2021.

15. Group SSCobotSBR. Risk of fatal coronary heart disease in familial hypercholesterolaemia. Bmj. 1991;303(6807):893-896.

16. Austin MA, Hutter CM, Zimmern RL, Humphries SE. Genetic causes of monogenic heterozygous familial hypercholesterolemia: a HuGE prevalence review. Am J Epidemiol. 2004;160(5):407-420.

17. Williams RR, Hunt SC, Schumacher MC, et al. Diagnosing heterozygous familial hypercholesterolemia using new practical criteria validated by molecular genetics. In. Vol 72: The American Journal of Cardiology; 1993:171-176.

18. Jackson CL, Keeton JZ, Eason SJ, et al. Identifying Familial Hypercholesterolemia Using a Blood Donor Screening Program With More Than 1 Million Volunteer Donors. JAMA Cardiol. 2019;4(7):685-689.

19. Ahmad ZS, Andersen RL, Andersen LH, et al. US physician practices for diagnosing familial hypercholesterolemia: data from the CASCADE-FH registry. J Clin Lipidol. 2016;10(5):1223-1229.

20. deGoma EM, Ahmad ZS, O'Brien EC, et al. Treatment Gaps in Adults With Heterozygous Familial Hypercholesterolemia in the United States: Data From the CASCADE-FH Registry. Circ Cardiovasc Genet. 2016;9(3):240-249.

21. Saloner B, Wilk AS, Levin J. Community Health Centers and Access to Care Among Underserved Populations: A Synthesis Review. Med Care Res Rev. 2020;77(1):3-18.

22. Hopkins PN, Stephenson S, Wu LL, Riley WA, Xin Y, Hunt SC. Evaluation of coronary risk factors in patients with heterozygous familial hypercholesterolemia. Am J Cardiol. 2001;87(5):547-553.

23. Harada-Shiba M, Arai H, Okamura T, et al. Multicenter study to determine the diagnosis criteria of heterozygous familial hypercholesterolemia in Japan. J Atheroscler Thromb. 2012;19(11):10191026.

24. Pijlman AH, Huijgen R, Verhagen $\mathrm{SN}$, et al. Evaluation of cholesterol lowering treatment of patients with familial hypercholesterolemia: a large cross-sectional study in The Netherlands. Atherosclerosis. 2010;209(1):189-194.

25. Mundal L, Sarancic M, Ose L, et al. Mortality among patients with familial hypercholesterolemia: a registry-based study in Norway, 1992-2010. J Am Heart Assoc. 2014;3(6):e001236. 
26. CDC. National Diabetes Statistics Report. In:2020.

27. Climent E, Perez-Calahorra S, Marco-Benedi V, et al. Effect of LDL cholesterol, statins and presence of mutations on the prevalence of type 2 diabetes in heterozygous familial hypercholesterolemia. $S c i$ Rep. 2017;7(1):5596.

28. Joud H, Mohamed E, Mirza S, et al. Prevalence and management of diabetes among the uninsured: A multicenter study in Tampa Bay, FL. Diabetes Res Clin Pract. 2021;171:108560.

29. Jones LK, Sturm AC, Seaton TL, et al. Barriers, facilitators, and solutions to familial hypercholesterolemia treatment. PLoS One. 2020;15(12):e0244193.

30. Pearson GJ, Thanassoulis G, Anderson TJ, et al. 2021 Canadian Cardiovascular Society Guidelines for the Management of Dyslipidemia for the Prevention of Cardiovascular Disease in the Adult. Canadian Journal of Cardiology. 2021.

\section{Figures}

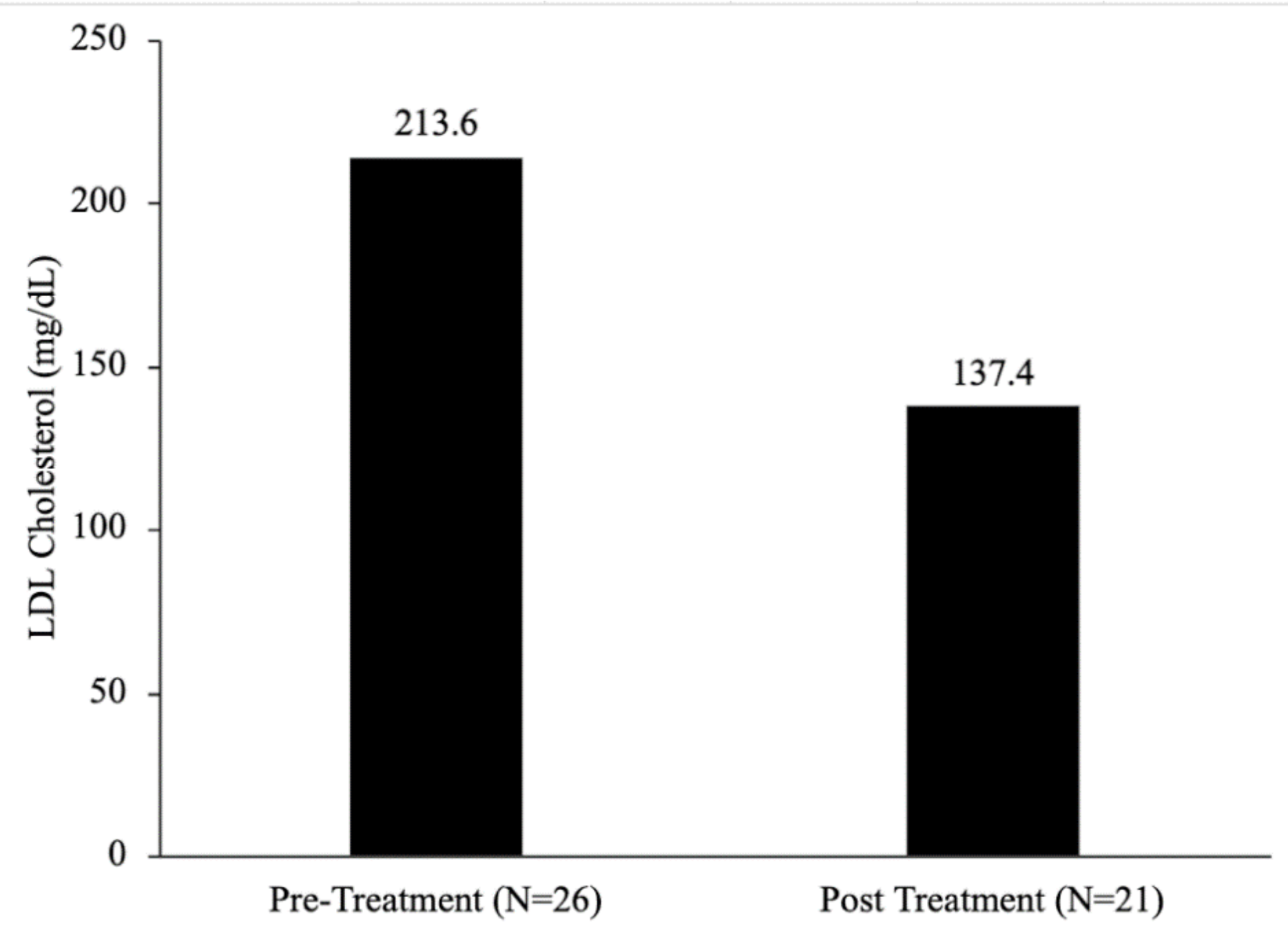

Figure 1

Mean LDL-cholesterol levels before and after statin therapy. 
The bars represent the mean values of LDL-cholesterol among the study population prior to and after treatment with a statin medication. The mean treatment period between untreated and treated values was approximately one year.

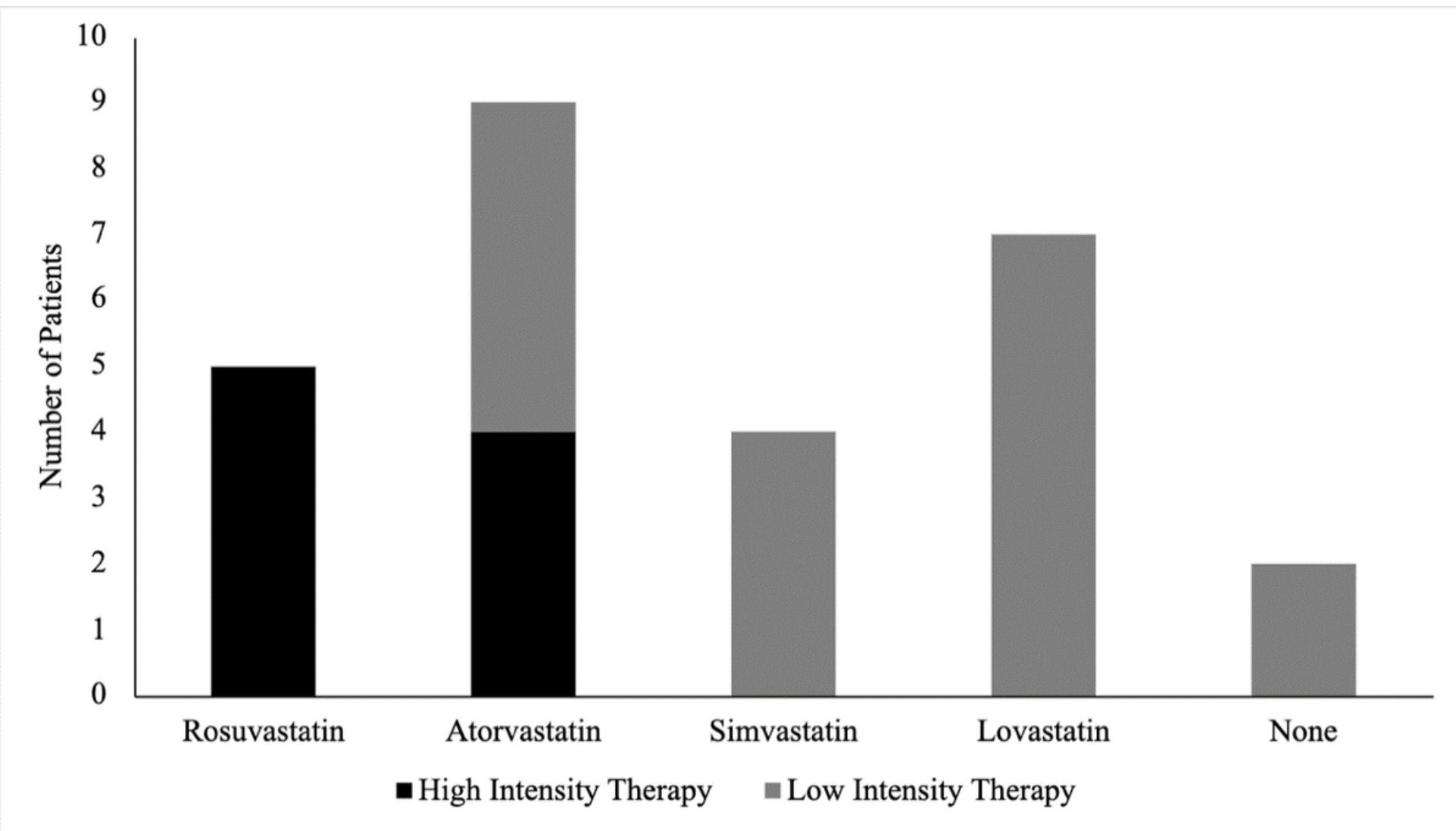

Figure 2

Statins prescribed to target patient population at NDSM clinic (LDL-C $\geq 190 \mathrm{mg} / \mathrm{dL})$

The bars represent the number of patients who were prescribed each form of therapy. Black bars represent therapy that is considered high intensity and grey bars indicate low or moderate intensity therapy. Total patients $n=27$.

\section{Supplementary Files}

This is a list of supplementary files associated with this preprint. Click to download.

- Supplementarymaterial.docx 